\title{
Top Cross Section and properties measurements at LHC
}

\author{
Luca Fiorini ${ }^{* \dagger}$ \\ Institut de Física d' Altes Energies (IFAE), Bellaterra, 08193 - Barcelona, Spain \\ E-mail: fiorinieifae.es
}

The study of the top quark phenomena will be a fundamental element of the early physics program at the Large Hadron Collider (LHC). The extremely high energy of the LHC proton-proton collisions and the large top quark production may provide an improved reach in several top quark measurements. We will show the expected performance of the ATLAS and CMS experiments focusing on the early measurements of the top pair and single top production. The LHC prospects for the measurement of the $V_{t b}$ element of the Cabibbo-Kobayashi-Maskawa (CKM) from top pairs and single top will be discussed. We will describe the LHC potential for the study of the top quark properties and physics beyond the Standard Model (SM) in the top quark sector. The sensitivity of the ATLAS and CMS experiments will be analyzed under different scenarios of LHC center of mass energy and integrated luminosity.

European Physical Society Europhysics Conference on High Energy Physics, EPS-HEP 2009,

July 16 - 222009

Krakow, Poland

\footnotetext{
* Speaker.

† On behalf of ATLAS and CMS collaborations. I would like to thank M. Bosman, R. Hawkings, C. Campagnari and T. Christiansen for their comments and useful discussions.
} 


\section{Introduction}

The determination of the $t \bar{t}$ production cross section is one of the first SM measurements that will be performed once the Large Hadron Collider (LHC) will start collisions at the end of 2009. At a luminosity of $10^{32} \mathrm{~cm}^{-2} \mathrm{~s}^{-1}$ and a proton beam energy of $5 \mathrm{TeV}$, a few weeks will be sufficient to collect a sample large enough to check the predictions of the SM [1].

The interest for the top quark physics more than 10 years after its discovery is due to various reasons: its large Yukawa coupling with the Higgs field grants it a special role in New Physics models; due to its abundant production at the LHC, it is well suited as a data-driven calibration tool; top decays, characterized by undetected neutrinos escaping the detector and by jets and leptons of high transverse momentum are an important background in the searches for the Higgs boson and physics beyond the SM. The determination of the top quark production rate and the detailed study of its properties will be stringent tests of the validity of the SM and will eventually reveal important details of the Electro-weak symmetry breaking mechanism.

While finalizing the commissioning of the detector, the ATLAS [2] and CMS [3] collaborations performed detailed studies of the various aspects of top quark physics using Monte Carlo simulations. The analyses tested the existing methods employed in TeVatron experiments and considered a large number of variants and extensions of the previous ideas. This paper will present a brief review of the top quark physics techniques and analyses studied by LHC experiments.

\section{Top Quark Production Measurements}

Fig. 1 shows the leading order diagrams of the $t \bar{t}$ production process: the production rate at LHC will exceed the TeVatron rate by about two orders of magnitude [4], passing from $\sigma(t \bar{t})^{\text {approx }} N N L O=$ $7.5 \pm 0.5 \mathrm{pb}$ at $1.96 \mathrm{TeV}$ to $\sigma(t \bar{t})^{\text {approx }} N N L O=401 \pm 25 \mathrm{pb}$ at $10 \mathrm{TeV}$ and $\sigma(t \bar{t})^{\text {approx }} N N L O=$ $883 \pm 45 \mathrm{pb}$ at $14 \mathrm{TeV}$. As the background will increase only by about a factor 10 from TeVatron to LHC, ATLAS and CMS will have the opportunity to measure the top properties with high statistics and low background contamination. Fig. 2 shows the the leading order diagrams of the single top production diagrams, that will also be boosted at LHC from about $3.5 \mathrm{pb}$ to $320 \mathrm{pb}$ (at $14 \mathrm{TeV}$ ).

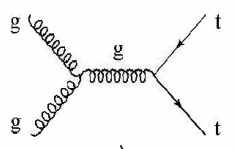

a)

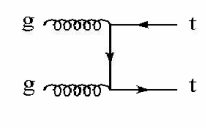

b)

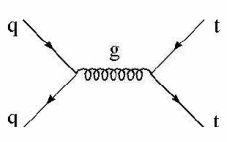

c)

Figure 1: Lowest level diagrams of the $t \bar{t}$ production. Gluon scattering processes, a) and $\mathrm{b})$, are the dominant processes at LHC, while quark scattering, process $\mathrm{C}$ ), is the dominant one at TeVatron energies.

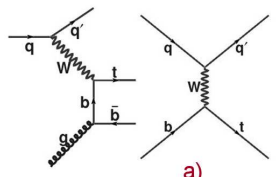

a)

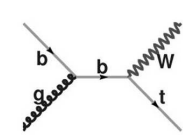

b)

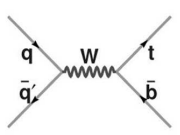

c)
Figure 2: Diagrams of single top production at the lowest level: a) t-channel, b) Wt associated production, C) s-channel.

ATLAS and CMS both plan to measure the $t \bar{t}$ cross section with early LHC data.

One of the CMS studies investigated the feasibility of the measurement with $10 \mathrm{pb}^{-1}$ of data in the di-lepton channel[5]. The event selection requires 2 isolated leptons (either muon or electron) and 2 jets plus missing transverse energy (MET). For a center of mass energy of $10 \mathrm{TeV}$ a statistical 
uncertainty of $15 \%$ and a systematic uncertainty of $10 \%$ are expected. The uncertainty on the luminosity measurement is expected to be of $10 \%$. Fig. 3 shows the expected number of events as a function of the number of jets. The $t \bar{t}$ signal for $\mathrm{N}_{\text {jets }} \geq 2$ is clearly standing over a small remaining background.

An ATLAS study investigated the expected accuracy of the $t \bar{t}$ cross-section measurement in the top single lepton channel for an accumulated statistics of $200 \mathrm{pb}^{-1}$ at $10 \mathrm{TeV}$ of center of mass energy [1]. One of the proposed analyses employs a robust selection requiring an isolated tight lepton (either muon or electron) and 4 jets in the final state. Fig. 4 shows the reconstructed hadronic top mass fitted with a template distribution of the signal from Monte Carlo (MC) simulation and a distribution of the main backgrounds obtained with data-driven techniques. The expected uncertainties are $6 \%_{(\text {stat })}, 15 \%_{(\text {syst })}$ and $20 \%_{(\text {lumi })}$.

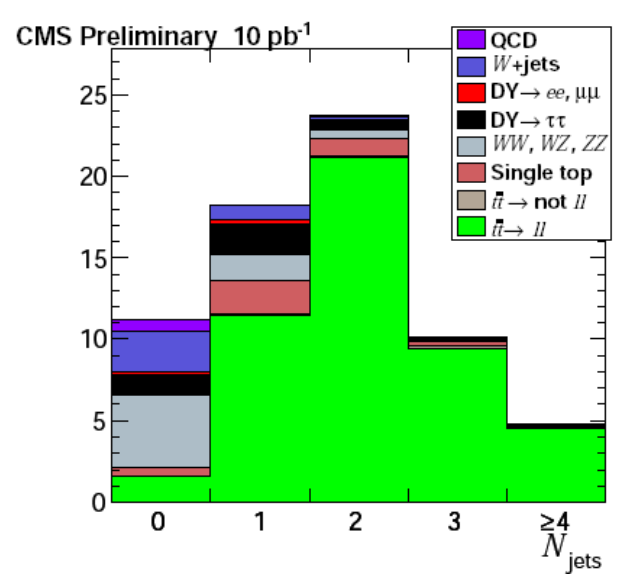

Figure 3: CMS number of expected events as a function of the number of jets in the $t \bar{t} 1$ muon-1 electron final state for $10 \mathrm{pb}^{-1}$. This final state is characterized by very low background contamination from Drell-Yan process.

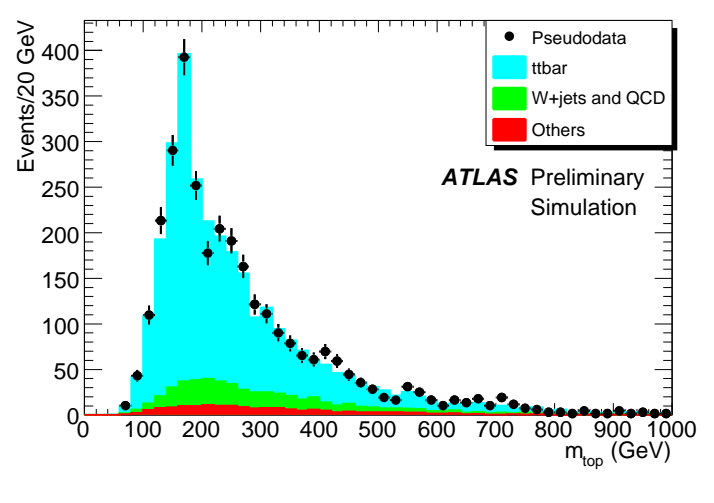

Figure 4: ATLAS reconstructed hadronic top mass in the $t \bar{t}$ single muon channel. The distribution is fitted with a MC template distribution of the signal shape, while the $W+j e t s$ and $Q C D$ background shapes are established with a data driven technique.

ATLAS and CMS evaluated the prospects for the measurement of the single top cross section, a direct measurement of the $V_{t b}$ element of the CKM matrix. At $10 \mathrm{TeV}$ of center of mass energy and with $200 \mathrm{pb}^{-1}$ of integrated luminosity, CMS expects to measure the t-channel cross section with an uncertainty of [6]:

$$
\frac{\Delta \sigma}{\sigma}= \pm 35 \%_{(\text {stat })} \pm 14 \%_{(\text {syst })} \pm 10 \%_{(\text {lumi })}
$$

The analysis relies on a template fit of the angle between the forward jet and the lepton in the center of mass frame. This kinematic variable yields a good signal discrimination and systematic checks proved that the result is robust.

ATLAS studied the t-channel cross section measurement in the $14 \mathrm{TeV}$ scenario with an integrated luminosity of $1 \mathrm{fb}^{-1}$. The analysis is based on a multivariate Boosted Decision Tree (BDT) technique. The expected accuracy is [7]:

$$
\frac{\Delta \sigma}{\sigma}= \pm 6 \%_{(s t a t)} \pm 22 \%_{(\text {syst }+ \text { lumi })}
$$




\section{Top Quark Properties}

With LHC providing more luminosity after the initial period of data taking, it will be possible to study the top quark properties with more precise measurements. One of the first measurements to perform will be the determination of the $t \rightarrow W b$ branching ratio, that is a measurement of the $V_{t b}$ element of the CKM matrix. CMS [8] expects to measure the $\mathrm{BR}(t \rightarrow W b)$ with $250 \mathrm{pb}^{-1}$ of data at $10 \mathrm{TeV}$ with a relative uncertainty of $10 \%$ dominated by the systematic contributions.

An interesting property that ATLAS expects to measure with $1 \mathrm{fb}^{-1}$ of data is the $t \bar{t}$ spin correlation predicted by the SM, that becomes particularly relevant in the case that a $t \bar{t}$ resonant state is discovered. The expected accuracy is of $11 \%($ stat $)$ and $9 \%($ syst $)$.

A $5 \sigma$ sensitivity to flavour changing neutral current (FCNC) processes in the top decays has been estimated by ATLAS and CMS collaborations [7] [9]. It is of $\sim 10^{-2}$ for the $\mathrm{BR}(t \rightarrow Z q)$ with 1 $\mathrm{fb}^{-1}$ of data (ATLAS) and $\sim 1.5 \cdot 10^{-3}$ with $10 \mathrm{fb}^{-1}$ (CMS). For the $\mathrm{BR}(t \rightarrow \gamma q)$ it is $\sim 10^{-2}$ with $1 \mathrm{fb}^{-1}$ of data (ATLAS) and $\sim 10^{-3}$ with $10 \mathrm{fb}^{-1}$ (CMS).

\section{Conclusions}

A brief overview of the expectations of the top quark measurements at LHC was given. The LHC will soon start the first proton-proton collisions at a center of mass energy never reached so far. ATLAS and CMS have detailed plans for analyzing the very large sample of top quarks that will be produced. The MC studies carried-out by the two collaborations show that the top quark production can be observed in the early stage of data taking and that top quark properties sensitive to new physics processes are also likely to be measured in the first period of LHC collisions.

\section{References}

[1] ATLAS Collaboration, "Prospects for the Top Pair Production Cross-section at $\sqrt{s}=10 \mathrm{TeV}$ in the Single Lepton Channel in ATLAS" [ATL-PHYS-PUB-2009-0 87].

[2] G. Aad et al., "The ATLAS Experiment at the CERN Large Hadron Collider" JINST 3 (2008), S08003.

[3] CMS Collaboration, "The CMS experiment at the CERN LHC" JINST 3 (2008), S08004.

[4] S. Moch, P. Uwer, "Theoretical status and prospects for top-quark pair production at hadron colliders" Phys. Rev. D 78 (2008), 034003 [arXiv: 0804 .1476].

[5] CMS Collaboration, "Expectations for observation of top quark pair production in the dilepton final state with the early CMS data at $\sqrt{s}=10 \mathrm{TeV}$ " [CMS PAS TOP-09-002].

[6] CMS Collaboration, "Prospects for the measurement of the single-top t-channel cross section in the muon channel with $200 \mathrm{pb}^{-1}$ of CMS data at $10 \mathrm{TeV}^{\prime}$ [CMS PAS TOP-09-005].

[7] ATLAS Collaboration, "Expected Performance of the ATLAS Experiment : Detector, Trigger and Physics" CERN-OPEN-2008-020 [arXiv:0901.0512].

[8] CMS Collaboration, "Probing the heavy flavor content of the tt dilepton channel in proton proton collisions at sqrt(s) $=10 \mathrm{TeV}$ " [CMS PAS TOP-09-001].

[9] CMS Collaboration, "Physics TDR II: Physics Performance", J.Phys. G34 (2007), 995-1579. 